\title{
Japanese nurses' perception of their experiences caring for culturally diverse patients: Comparison with Korean nurses
}

\author{
Noriko Kuwano $^{1 *}$, So Woo Lee ${ }^{2}$, Soong-nang Jang ${ }^{3}$ and Hiromi Fukuda ${ }^{1}$ \\ ${ }^{1}$ Oita University of Nursing and Health Sciences, Megusuno, Oita City, Oita, Japan \\ ${ }^{2}$ Emeritus Professor of Seoul National University, College of Nursing, Seoul National University, Daehak-ro, Jongno-gu, Seoul, Republic of Korea \\ ${ }^{3}$ Red Cross College of Nursing, Chung-Ang University, Nursing Science Research Institute, Chung Ang University, Heukseok-ro Dongjak-gu Seoul, South Korea
}

\begin{abstract}
Purpose: The purpose of this study was to explore Japanese nurses' perceptions of their experiences caring for culturally diverse patients through a comparison with Korean nurses.

Methods: A qualitative exploratory design with a thematic analysis approach was used to collect and analyze data. Using purposive sampling, 15 nurses (9 Japanese nurses, 6 Korean nurses) who provided nursing care to culturally diverse patients within the past year were selected. Data were gathered using semi-structured interviews. The six steps of Braun and Clarke were followed for data analysis.

Results: Based on the data analysis, the following three themes were identified: challenges regarding the care of culturally diverse patients; important components regarding the care of culturally diverse patients; and deficiencies in nursing education for culturally congruent care.

Conclusion: In the present study, Japanese nurses had less experience and provided fewer descriptions than Korean nurses regarding changes in thinking gained through experience caring for culturally diverse patients, and they also expressed feeling anxiety when providing care for such patients. This suggests that organizational support for culturally-based care should be promoted in Japan. Furthermore, our findings also suggest that education/training programs should be offered in order to enhance the cultural competency of Japanese and Korean nurses.
\end{abstract}

\section{Introduction}

In a now classic definition, culture is defined as learned, shared, and transmitted knowledge of values, beliefs, and norms of a group that influences behavior [1]. Based on this definition, the influence of culture can be seen in individual and group health-seeking behaviors and attitudes toward illness. Japan is becoming an increasing culturally diverse country, it is essential that nurses be adequately prepared for and able to work with patients in a way that recognizes and respects this diversity [2]. Cultural competency has been identified as a core competency in globalized society by the National League for Nursing [3], and thus the development of cultural awareness and the acquisition of knowledge regarding cultural diversity should be emphasized among both clinical nurses and nursing students [4].

Japan, a historically mono-cultural society, has been progressively transitioning into one that is more culturally diverse. This is due in part to the large numbers of Japanese descendants who have repatriated to Japan since the revision of the Immigration Control Law in 1990 [5]. In addition, the Japanese government initiated strategies such as the "300,000 International Students Plan" [6] and "Points-based Preferential Immigration Treatment for Highly Skilled Foreign Professionals" [7] to strengthen international competitiveness and bring in younger professionals to support Japanese society. As a result of these efforts, the numbers of foreign residents in Japan have been steadily increasing. Consequently, nurses are presented with the challenge of caring for the increased numbers of culturally and linguistically diverse patients and their families who inevitably visit hospitals. In fact, many nurses have already been exposed to more culturally diverse patients without being adequately prepared [8]. Therefore, concern about the adequate provision of nursing care for culturally diverse patients has recently been increasing in Japan.

Culturally congruent care takes different forms based on how cultural backgrounds are characterized by health care providers, and the content of culturally congruent care varies depending on how well a culture is understood [9]. Furthermore, nurses' previous experiences in caring for different cultural groups enhance their ability to provide appropriate cultural care [10]. From this perspective, to improve the quality of care provided by Japanese nurses to foreign patients, it is important to understand nurses' perceptions of their previous experiences in caring for patients with diverse cultural backgrounds.

Korea is Japan's geographically closest neighbor, and although both countries have a similar socioeconomic background, their health care environments in relation to the treatment of ethnically diverse patients are markedly different. The Korean government has made great efforts to rapidly develop global health care over the past decade [11]. In 2009, the Korean Ministry of Health and Welfare established

Correspondence to: Noriko Kuwano, Lecturer, Oita University of Nursing and Health Sciences, 2944-9 Megusuno, Oita City, Oita, Japan, Tel: +81-97-5864487; Fax: +81-97-586-4388; E-mail: kuwano@oita-nhs.ac.jp

Key words: cultural competency, cross cultural comparison, cultural diversity, thematic analysis

Received: August 20, 2017; Accepted: September 19, 2017; Published: September 22, 2017 
the Korean Global Healthcare Association [12] to promote medical tourism and the overseas expansion of Korean hospitals. To aid this effort, medical interpretation services were established in several large hospitals [11]. A previous study on identifying employment experiences of nurses caring for foreign patients in Korea summarized that Korean nurses explained their experiences as five categories; 1) Awesome challenges; 2) Laborious dealing with barriers; 3) Acceptance of cultural differences; 4) Complete concentration of personal energy; 5) Taking a powerful step towards tomorrow [13]. Comparing the differences between Korean and Japanese nurses in relation to caring for ethnically diverse patients should enable a clearer understanding of Japanese nurses' perceptions of their previous experiences.

Therefore, the aim of this study was to explore Japanese nurses' perceptions of their experiences of caring for culturally diverse patients through a comparison with those of Korean nurses. Through this comparison, this study hopes to further clarify the characteristics of Japanese nurses regarding the way they perceive and interpret their experiences caring for culturally and linguistically diverse patients. The results are expected to serve as basic reference material for further research for the formulation of policy regarding the provision of culturally congruent care by Japanese nurses.

\section{Methods}

\section{Aim and design}

The aim of this study was to investigate Japanese nurses' perceptions of their experiences caring for patients with diverse cultural backgrounds through a comparison with Korean nurses. A qualitative design with a thematic analysis approach was used for collecting and analyzing data.

\section{Study setting}

The study settings for the present study comprised general hospitals and community health center in Japan, and general hospitals in Korea. All settings in both countries were located in areas with high concentrations of foreign residents.

\section{Participants}

A total of 15 nurses (9 Japanese nurses, 6 Korean nurses) were selected through purposive sampling. The initial a couple of participants were recruited by supervisor recommendation, and after that, the researcher introduced a snowballing approach whereby those already recruited assisted the researcher in finding further participants who met the selection criteria. Sampling continued until data saturation was reached. Inclusion criteria were as follows: (a) participants had to work as a professional nurse; (b) participants had to have provided nursing care to at least one non-Japanese/non-Korean patient within the past year; (c) participants had to have been employed at the same hospital for at least one year; and (d) participants had to have given their consent to participate in the study.

\section{Data collection}

Data were gathered by in-depth, semi-structured, face-to-face interviews that were conducted between 2012 and March 2013. Each interview lasted between 40 and 60 minutes. The first author carried out all interviews in Japan and four of the six interviews in Korea. The other two interviews in Korea were conducted by the co-author. Interview guidelines were developed to ensure congruence between the two interviewers. All interviews took place in a calm environment in the participants' or researcher's offices. Interviews were audio-recorded and transcribed from the participants' native language (Japanese, Korean, or English) into Japanese after each session. All translations from Korean to Japanese were performed by a Japanese scholar living in Korea who was fluent in Korean. The accuracy of the transcriptions was checked by two researchers familiar with the study who were also acquainted with the Korean language and culture. The main interview questions regarded the following topics: the circumstances of caring for a culturally and linguistically diverse patient, and what the nurse felt at the time of care provision; what the nurse thinks as a nursing professional when caring for such patient; and experiences concerning learning about providing care for culturally diverse patients. We used probing questions during the interviews to obtain the clearest responses possible.

\section{Ethical considerations}

The study was approved by institutional review board (IRB approval No.659). Documents for participants were prepared in both Japanese and Korean. All participants were given information about the study, including the aims of the research and the personal details of the researchers. Written informed consent was obtained from each participant before the interviews. All nurses were also informed that their participation in the survey was voluntary, that they were free to withdraw at any time without penalty, that their anonymity would be preserved, and that the confidentiality of the collected data was guaranteed. They were also assured that all records would be deleted at the conclusion of the study. In addition, corporate approval was obtained from each hospital or nursing department director.

\section{Data analysis}

A thematic analysis approach was used to analyze the data. Thematic analysis is a method for identifying, analyzing, and reporting patterns (themes) within qualitative research data. It can be an essentialist or realist method, which describes the experiences, meanings, and the realities of participants [14]. This method is typically used for health research projects, particularly those that are simply descriptive or aim to describe key issues of particular groups or individuals [15]. We followed the six phases of analysis described by Braun and Clarke [14] to analyze data in this study. In the first phase, the transcribed interviews were read several times to familiarize ourselves with the data. In phase 2, initial codes were generated from the data. First, initial codes were produced for as many potential themes/patterns as possible. Relevant codes were then identified from among these codes. In phase 3 , we searched for themes by analyzing the codes and considering how different codes could be combined to form an overarching theme. In phase 4 , all themes were reviewed. In this phase, researchers identified both themes and sub-themes by coordinating between codes, and then confirmed the initial themes. This enabled us to then generate a thematic map for phase 5. In phase 6 , the final phase, we defined and named the confirmed themes. In this phase, researchers further refined the themes by reviewing the entire process of data analysis for producing the report.

\section{Rigor}

To examine the trustworthiness of our qualitative data and analysis, we employed several different strategies according to Lincoln and Guba's evaluation criteria [16]. In collecting the data, accurate transcriptions and translations were performed. During the interview process, if the translator had any difficulty with a translation, the researcher would provide alternative equivalent terms and collaborate with the translators to make a final decision on the terms and their related 
meanings. In order to attain credibility, researchers took the time to analyze emerging data, and in case of Japanese participants, employ member checking. Japanese participants confirmed authenticity of the data and categories based on main researcher's proposal. In case of Korean participants, interpreter and co-researcher supported to confirm the data and categories. Throughout the process of analysis, ongoing discussion was conducted with two supervisors, Japanese and Korean professor who had been worked both in Japan and Korea.

\section{Results}

The Japanese participants ranged in age from 20 to 49 years and Korean participants from 20 to 39 years, and they were all female (Table 1). Four of the six Korean nurses belonged to the international ward, which accept foreign patients almost daily, mainly medical tourists from various countries such as Mongolia, the United Arab Emirates, the United States, and the Russian Federation. Only one of the nine Japanese nurses belonged to an international ward and cared for foreign patients almost daily; the patients' backgrounds were foreign residents who were working in Japan or their families, and tourists. The others cared foreign patients just a few times each month.

The following three major themes emerged from data analysis: "challenges regarding the care of culturally diverse patients"; "important components regarding the care of culturally diverse patients"; and "deficiencies in nursing education for culturally congruent care". The first theme included the following three sub-themes: emotional reactions of nurses (Japan, Anxiety; Korea, Pressure); barriers regarding the care of culturally diverse patients; and ideas gained through experiences. The second theme included the following four sub-themes: understanding patients with different cultural backgrounds; improving communication skills; open-mindedness; and demonstrating typical provision of care (only Japan). The third theme had no sub-themes. The following interpretations, accompanied by direct quotes from the participants, demonstrate these three themes.

\section{Challenges regarding the care of culturally diverse patients}

This theme described the participants' perceptions regarding their past experiences in caring for culturally and linguistically diverse patients. The three sub-themes are emotional reactions of nurses; barriers regarding the care of culturally diverse patients; and ideas gained through experiences.

Emotional reactions of nurses (Japan, Anxiety; Korea, Pressure): Both Japanese and Korean nurses perceived difficulties in providing care to culturally and linguistically diverse patients. Japanese nurses felt uneasy or experienced feelings of anxiety in relation to communication, relationships between nurses and patients, and effectiveness of their care. Korean nurses expressed feeling pressure to live up to patients' expectations as opposed to anxiety.

\section{A Japanese participant stated:}

"Whenever I was in contact with a foreign patient, I always felt anxious about things like whether I was offending them with just some small nuance, or whether I was managing to get my meaning across." (Japan)

Demonstrating another perspective, a Korean nurse said, "I felt a little pressure at first. I wanted to avoid the situation if possible, but I was dealing with a patient, so I had to accept it." (Korea)

Barriers for the care of culturally diverse patients: The language barrier was perceived as a major problem in relating to patients by nurses in both Japan and Korea. However, in contrast to Korean nurses, Japanese nurses described more clearly an insufficiency of professional interpretation services in their work setting. For example, one Japanese nurse commented:

"I can't explain the reason for care, or the results it will give. Just giving an intravenous drip is really tough when I think about how anxious the patient feels." (Japan)

\section{Another said:}

"The interpreters are not medical professionals, so sometimes what I am saying is not properly explained to the patient. This means that I can't always give the patient the sort of supplementary explanations I would give a Japanese patient." (Japan)

\section{A Korean participant shared another aspect of this problem:}

"The biggest thing is the language problem. I can speak English, but if the patient is a non-English-speaker, we have to speak through a third person, which makes it difficult to build a rapport." (Korea)

In addition to the language barrier, almost all nurses in both countries had been involved in a care situation in which they experienced difficulties related to the different cultural and social backgrounds of the patients. These differences included medical systems, family relationships, views concerning the roles of nurses, medical literacy, and attitudes of patients toward the pain associated with medical procedures. The participants reported also that they encountered problems in care due to different religious and dietary customs.

\section{A Japanese nurse illustrated this point:}

"With religious customs, there are very detailed requests and questions about dietary restrictions. The nurse has to come between the patient and the nutrition department, which can be tough." (Japan) Another said:

"I think there are differences in medical literacy. If you mention cancer, patients from some countries think that the only choice is to cut it out or die. They don't understand the other options." (Japan)

\section{A Korean participant concurred:}

"One patient had a special time for prayer, and any treatment, tests, or care had to be given at other times. I felt that we needed to have a thorough awareness of prayer time." (Korea)

Another brought up a cultural difference in health care:

"Korean people can have a free medical checkup once a year. However, in some countries, they don't even have the concept of health checkups. I don't think they have the chance for a medical checkup until they actually get ill. When I recommend a health checkup, I get a reaction as if to say, 'Why should I do that?"' (Korea)

Ideas gained through experiences: Most Japanese nurses had fewer experiences caring for culturally diverse patients than Korean nurses. However, one Japanese nurse expressed that the important issue is to realize our own barriers and stereotypes. A Japanese nurse reflected:

"Many people think that there is a language barrier with those from other countries, but my feeling is that you can manage about $70 \%$ through non-verbal communication. I don't think it's the language barrier-the first thing I would like people to get rid of is the emotional barrier." (Japan) 
Half of the Korean nurses described changes in their perceptions regarding the care for culturally diverse patients after several years of experience. Through experience, they started to become interested in learning more about cultural diversity and they gradually began to understand more about different cultures. They realized that understanding patients' cultural backgrounds enabled smoother communication and more effective care. They also perceived that caring for culturally diverse patients represented a precious opportunity to learn about how to provide world-class patient care. Moreover, one experienced Korean nurse reported that open-mindedness, not language skills, was the most important factor for providing quality care, saying:

"I always used to believe that verbal communication was the most important thing and the biggest challenge, but I have changed my way of thinking. Now I think that the most important thing is to keep an open mind, and it is important to understand patients from around the world and to build an emotional rapport. For example, even if doctors or nurses are fluent in English or Japanese or Russian or whatever, they won't be able to understand foreign patients if they have closed minds and just focus their attitudes and ideas on Korean patients." (Korea)

Both Japanese and Korean nurses voiced difficulties in caring patient with divers culture, in addition, more experienced nurses emphasized the importance of realizing their own barrier and changing themselves rather than verbal communication.

\section{Important components regarding the care of culturally diverse patients}

This theme describes important care components regarding the knowledge, skills, and attributes that participants believed were required by nurses to provide culturally competent care. This theme includes four sub-themes, three of which were common to both Japanese and Korean nurses. The other sub-theme was derived from Japanese nurses only. The four sub-themes are: understanding patients with different cultural backgrounds; improving communication skills; open-mindedness; and demonstrating typical provision of care.

Understanding patients with different cultural backgrounds: Many participants commented that understanding patients with different cultural backgrounds is important in order to provide appropriate care, and that nurses themselves should be culturally sensitive so that they can be aware of differences between patients' cultures and their own. Both Japanese and Korean nurses commented as follows:

"Different cultures, different values-when working with a patient, you have to understand what kind of culture the patient's country has. We really have to understand what the customs of other countries are, and we can't let fuzzy thinking get in the way of this." (Japan)

"The most important thing is to understand the patient's background, which includes the cultural background, so that you can treat every individual with the same respect that you give when caring for a Korean patient." (Korea)

This sub-theme was congruent with the previous theme of barriers to care, especially language and cultural barriers.

Improving communication skills: Participants also referred to the necessity of improving both their verbal and non-verbal communication skills. One Japanese nurse stated:

"If you can't communicate verbally, this may increase the chance of a misunderstanding, so I think you need a certain level of linguistic ability in order to be able to provide safe care.” (Japan)
Another said:

"The way you communicate is important. Even if you speak different languages, the other person will listen if you make the effort to get your meaning across by using gestures or breaking up what you want to say into single words. The important thing is to show that you are trying to communicate, and not to give up." (Japan)

\section{A Korean nurse concurred:}

"I think communication is important. Patients often feel anxious, so you have to explain things as carefully as possible." (Korea)

Open-mindedness: One Japanese and one Korean nurse who each had a wealth of experience caring for culturally diverse patients commented on the importance of keeping an open mind and eliminating stereotypes. The Japanese nurse said:

"Patients from different countries simply have different cultural backgrounds and different languages, but many people feel that there are emotional barriers. There are also a lot of stereotypes. I think people should take a neutral standpoint, and not see foreigners in a negative way." (Japan)

\section{Her Korean counterpart said:}

"I think the misunderstandings that happen sometimes are the result of cultural differences. They are just the result of having a closed mind, so I think we should have more opportunities to study other cultures in detail. That would allow us to eliminate our own stereotypes." (Korea)

\section{Another Korean nurse said:}

"An open mind is the most important thing when caring for foreign patients. We have to understand patients from around the world and build an emotional rapport." (Korea)

Demonstrating typical provision of care: The final sub-theme in the care components theme was derived from interviews of Japanese nurses only. Japanese nurses thought about their ability to provide safe care with confidence, as this participant commented:

“The care is the same. I don't believe Japanese nursing staff provide a low level of care. I want to provide the nursing I am capable of without being timid." (Japan)

Three components; Understanding cultural background, keep trying to communicate both in verbally and non-verbally, and openmindedness were shared by two countries, however, only Japanese nurses mentioned about possibility of lower level nursing care for nonJapanese patient.

\section{Deficiencies in nursing education for culturally congruent care}

The third theme describes the participants' experiences of education in relation to culturally competent care. It includes what the participants had learned in their nursing education, what they thought about the necessity of specific education for culturally competent care, and topics about which they wished to learn more. In our study, we found that only one Japanese nurse had learned about health-related culture in an undergraduate nursing program. Two of the six Korean nurses had taken an undergraduate course on medical tourism; however, this course was primarily intended for aspiring travel agents, not health care providers. Three of the six Korean nurses reported that they had independently studied cultures of several different countries with a colleague. 


\section{A Japanese nurse mused:}

"The Economic Partnership Agreement is advancing, so there will probably be an increase not only in foreign patients, but also in nurses from other countries. We won't make any progress unless we get rid of the idea that only the Japanese way of perceiving things is normal." (Japan)

\section{In Korea, a focus on globalization is growing, as one nurse stated:}

"With increasing globalization, there will undoubtedly be a greater variety of patients from many more different countries than at present. To meet this challenge, we will need to provide one-on-one nursing tailored to individual patients. I think doing this will increase the level of patient satisfaction and increase the quality of medical care, so that our own satisfaction will also increase. There are a great many things that we need to study more." (Korea)

Almost all of the nurses in both countries commented that education for culturally competent care should be provided due to the clinical need to solve problems and provide high quality care. A Japanese nurse said, "I need to keep studying because there are areas of my work where I have difficulties." (Japan)

One nurse from Korea commented on her limited preparation for this role:

"There is a difference between nurses who studied global nursing at college, even if they didn't get credit for it, and those that didn't. There are two or three foreign people in our department, and I think we need to study international nursing. If I had studied just a little bit about cultural differences while at college, the initial difficulties wouldn't have been quite so bad. As it was, I just had to make the best of it. It was a situation I couldn't escape from even if I had wanted to, and if I had studied before I came here, I would have probably been a better nurse." (Korea)

Almost nurses in both countries had very few chance to study about culturally competent care, though they have strong motivation to study for better nursing. Three same themes were derived through interview from both countries, and there were differences between two countries in a couple of sub themes.

\section{Discussion}

In this study, Japanese and Korean nurses were asked about their perceptions regarding their experiences caring for culturally diverse patients. Numerous similarities were found in the components of perception identified by the Japanese and Korean nurses. Korean nurses also had more experience than Japanese nurses in caring for culturally diverse patients; however, both groups voiced many areas of difficulty or concern and a need for further education in this area. As several participants commented, as the first step, increasing awareness of cultural differences and respecting cultural diversity should be emphasized in nursing education in both countries.

Three themes resulted from the thematic analysis of interview data, highlighted in the following discussion.

\section{Challenges regarding the provision of care for cultur- ally diverse patients}

Examination of the first theme demonstrated the emotional reactions of nurses when they care for culturally diverse patients and barriers to providing appropriate care for such patients. Changes in thinking gained through experience were also demonstrated. Many of the Japanese nurses referred to their feeling in relation to their communication and relationship with patients and the effectiveness of their care as "anxiety," whereas Korean nurses referred to their feelings as "pressure" to live up to patients' expectations. The majority of patients for whom the Korean nurses provided care were medical tourists, and thus quality medical treatment was a primary consideration [17]. In Korea, medical tourism has been embedded in the culture, and its presence is continuously growing. On the other hand, the patients in Japan were primarily foreign residents who were working in Japan or their families. Medical tourism is still in the early stages in Japan. Differences not only in the cultural, but also in the social and economic backgrounds of patients, appeared to affect the emotional reaction of nurses when providing care.

The expression of emotions was also different between the two countries. In the present study, both Japanese and Korean nurses reported encountering difficulties caring for culturally diverse patients. The findings from this study support those from previous research concerning difficulties that nurses may feel when caring for culturally diverse patients [18-20]. Adachi, et al. [19] found that "communication problems", "interaction with a patient's family", "differences in culture and customs", "food", and "religious taboos" caused difficulties in nursing care for foreign patients. According to Maeno, et al. [20], nurses had difficulties caring for foreign patients due to the language barrier, cultural differences, and differences in health care systems. In this study, nurses from both countries perceived the language barrier as a major problem in relating to culturally and linguistically diverse patients. However, the deficiency of interpretation services by professionals was only mentioned by Japanese nurses. In Japan, only a few hospitals provide interpretation services, and these are volunteer-run and only offered for a limited number of languages [21]. This suggests that interpretation services should be supported at the organizational level.

The change of thinking gained through experience was another point that was mentioned by nurses. Experienced nurses in both countries focused on their own attitudes towards patients rather than the language barrier. Huang, Yates, and Prior [10] reported that nurses' previous experiences, both personally and with patients from diverse cultural backgrounds, played an important role in shaping their approach to care. In this study, nurses utilized their previous experiences caring for patients who had different cultural backgrounds to enhance their ideas about the provision of appropriate care. However, in the case of nurses who had less experience caring for such patients, they seemed to be unable to enhance their provision of culturally competent care.

\section{Important components regarding the care of culturally diverse patients}

The second theme highlighted components perceived as important regarding appropriate care for culturally diverse patients. The importance of improving the communication skills of nurses, and of understanding the cultural differences between patients and themselves, has been reported in a previous study. That study revealed components nearly identical to those identified in the present study. Language as a means of communication is fundamental for effective nurse-patient relationships, without which, nursing care is incomplete [18].

In this study, some nurses placed an emphasis on the importance of keeping an open mind and being aware of their own stereotypical assumptions in order to provide culturally appropriate care. Keeping an open mind is the willingness to learn about differences and treat 
each person as an individual in a way that can ultimately reinforce behavioral understanding of their culture [22]. However, in addition, this research identified the possibility that Japanese nurses sometimes have difficulty providing a typical level of care for culturally diverse patients.

\section{Deficiencies in nursing education for culturally congruent care}

In the third theme, the need for education/training for developing cultural competence, as well as deficiencies in nursing education/ training, were mentioned by almost all nurses from both countries. Several reasons why education was needed were mentioned. The most common reason was to learn the global standard of care, namely, how to provide world-class care for patients [17]. In addition, Japanese nurses mentioned that their aim was to cope with clinical problems. Korean nurses mentioned that their aim was to enhance their feeling of satisfaction and to eliminate their stereotypes.

Japanese nurses receive relatively little transcultural nursing education [23,24] and research preparation [25]. Due to its isolated geographical location and comparatively restrictive immigration policy [26], Japan has historically been considered a relatively homogeneous nation; therefore, transcultural nursing education and research preparation have seldom been viewed as necessities. Ono [23] investigated 20 fundamental nursing texts in Japan and found that only two of them mentioned "culture", and that only one briefly introduced Leininger's theory. Nonaka and Higuchi [25] indicated that few studies had been conducted in relation to cultural awareness, which is considered to be very important for the care of non-Japanese patients. Concerning Korean nurses, some of them had taken a course of medical tourism, even though it did not focus on actual nursing care. When a professional nurse perceives ignorance about cultural differences, they become anxious about being culturally inappropriate and causing affront [27]. Jirwe, et al. [27] indicated that although nurses tried to "do their best," they felt professionally disempowered, which created a disabling hesitancy and inertia in their practice. A lack of preparation might cause clinical nurses to be less able to provide appropriate care for foreign patients. Our results suggest that providing nurses with education programs is important for enhancing their cultural competency and improving future encounters (Figure 1 and 2).

The results of this study are limited in their generalizability due to a couple of factors. One limitation lies in the representativeness of the sample. Because of biased backgrounds of participants, such as age distribution and experienced/inexperienced in caring for culturally diverse patients, the results of this study cannot be extrapolated to all Japanese nurses or Korean nurses. Another limitation is related to the small sample size from each country. While a small sample size is normal for qualitative studies, further research is needed to ascertain whether our findings are more widely generalizable.

\section{Conclusion}

In the present study, Japanese nurses had less experience and provided fewer descriptions than Korean nurses regarding changes in thinking gained through experiences caring for culturally diverse patients. They also expressed feeling anxiety when providing care for such patients. One of the reasons for their anxiety could be the lack of organizational support in promoting care for culturally and linguistically diverse patients. Organizational support for culturallybased care should be promoted in Japan. Furthermore, our findings suggest that education/training programs should be offered in order to enhance the cultural competency of Japanese and Korean nurses. Providing culturally competent care for patients from diverse backgrounds who have a wide range of values and beliefs requires in-depth preparation. Therefore, to allow the provision of culturally competent care to patients by Japanese nurses, further research is needed to identify and clarify the scope of education and research necessary. It would be valuable to undertake a further study in the future to examine levels of cultural competence and requisite skills to provide care to foreign patients in diverse health care settings.
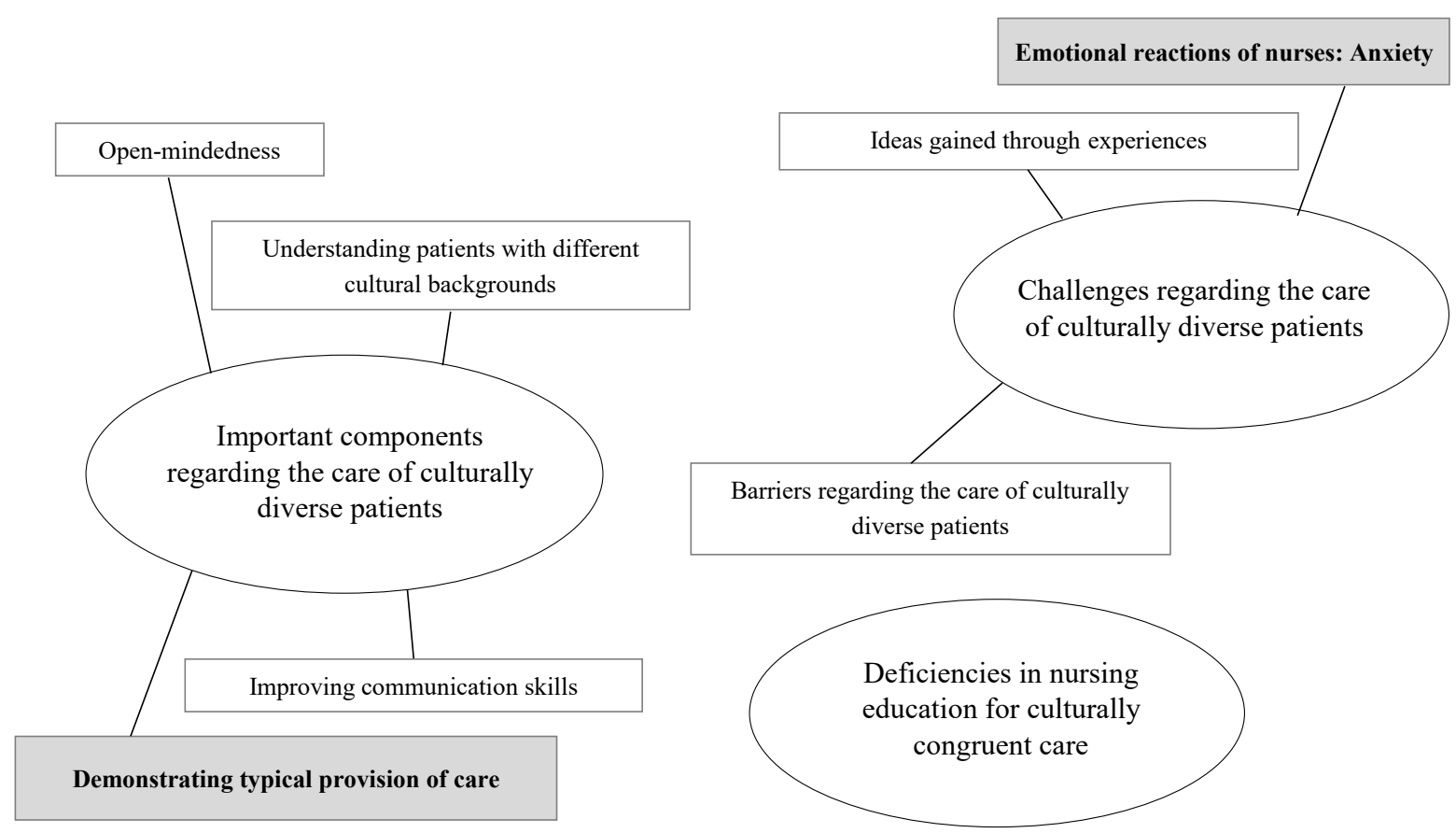

Figure 1. Developed thematic map of Japanese nurses, showing three main themes. 


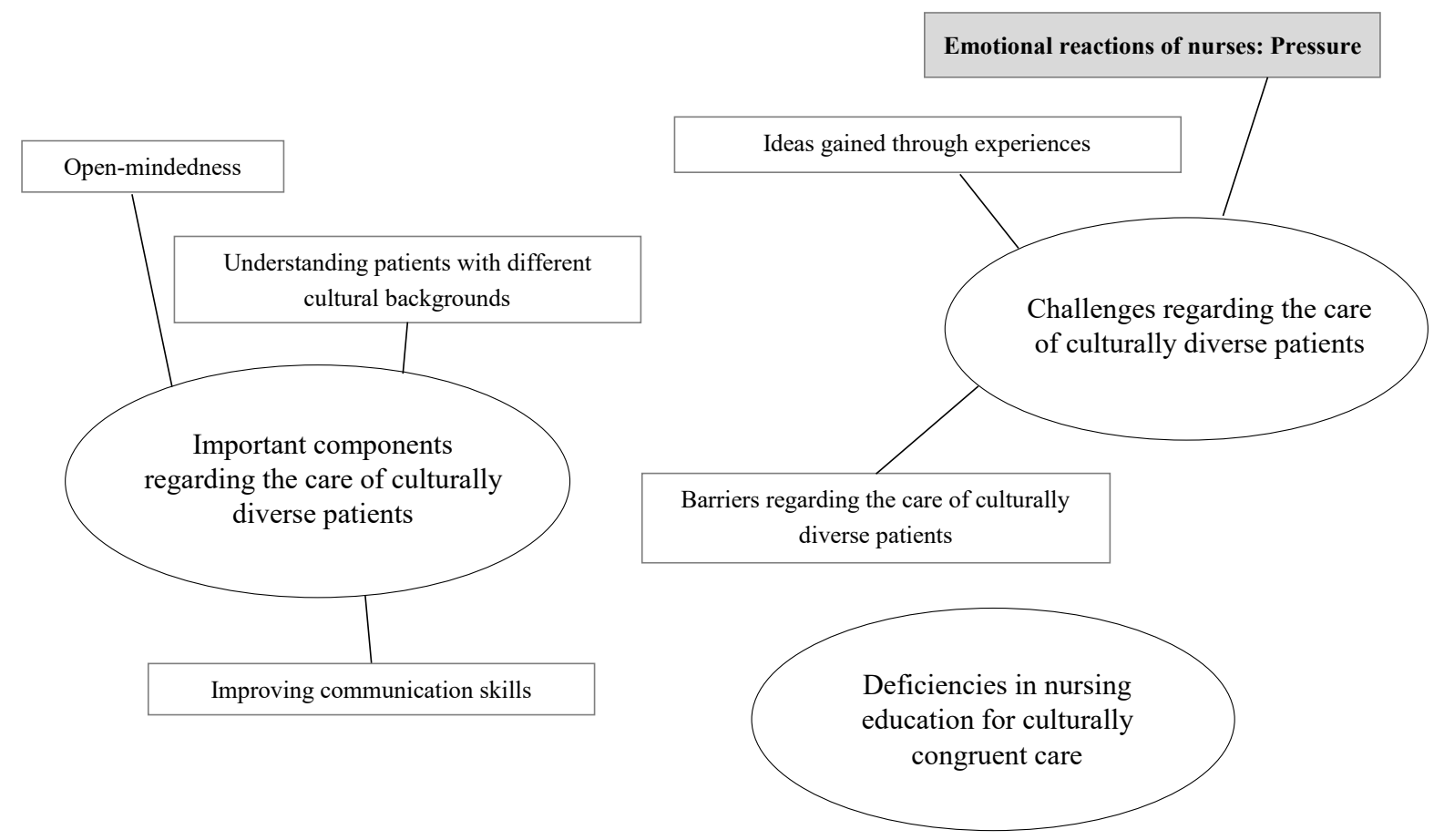

Figure 2. Developed thematic map of Korean Nurses, showing three main themes.

Table 1. Characteristics of the subject $(N=15)$

\begin{tabular}{|c|c|c|c|c|c|}
\hline Country & Age & Qualification & Setting & $\begin{array}{l}\text { Study opportunity at } \\
\text { school/at clinical }\end{array}$ & Interpretation service \\
\hline Japan & $40-49$ & $\mathrm{RN}$ & Hospital & No & No \\
\hline Japan & $20-29$ & $\mathrm{RN}$ & Hospital & No & No \\
\hline Japan & $40-49$ & $\mathrm{RN}$ & Hospital & No & No \\
\hline Japan & $30-39$ & MW & Hospital & No & Yes \\
\hline Japan & $30-39$ & $\mathrm{RN}$ & Hospital & No & Yes \\
\hline Japan & $40-49$ & $\mathrm{RN}$ & Hospital & No & Yes \\
\hline Japan & $40-49$ & PHN & Public Health Center & No & No \\
\hline Japan & $30-39$ & PHN & Public Health Center & Yes & No \\
\hline Japan & $30-39$ & PHN & Public Health Center & Yes & No \\
\hline Korea & $30-39$ & $\mathrm{RN}$ & Hospital & No & Yes \\
\hline Korea & $20-29$ & $\mathrm{RN}$ & Hospital & No & Yes \\
\hline Korea & $20-29$ & $\mathrm{RN}$ & Hospital & No & Yes \\
\hline Korea & $20-29$ & $\mathrm{RN}$ & Hospital & No & Yes \\
\hline Korea & $20-29$ & $\mathrm{RN}$ & Hospital & No & No \\
\hline Korea & $20-29$ & $\mathrm{RN}$ & Hospital & No & No \\
\hline
\end{tabular}

\section{Acknowledgments}

The authors gratefully acknowledge the participation of the nurses for their great contributions to this study, and Kathy Magilvy, PhD, RN, FAAN, who provided considerable knowledge to enhance the article.

This study was supported by a grant from the Ministry of Health, Labor and Welfare in Japan for the study (Grant No. 24659964).

My coauthors and I do not have any interests that might be interpreted as influencing the research.

\section{References}

1. Leininger M (2002) Transcultural Nursing: Concepts, Theories and Practices. ( $3^{\text {rd }}$ Edn) John Wiley \& Sons, New York p.47.

2. McClimens A, Brewster J, Lewis R (2014) Recognizing and respecting patients' cultural diversity. Nurs Stand 28: 45-52.
3. Diversity \& Inclusion toolkit (2009) National League for Nursing. Centre for Diversity and global initiatives.

4. Mareno N, Hart PL (2014) Cultural competency among nurses with undergraduate and graduate degrees: implications for nursing education. Nurs Educ Perspect 35: 83-88.

5. Japan Ministry of Justice (2014) Changes in the number of registered foreign nationals as of the end of 2013. Immigration Bureau.

6. Ministry of Education (2008) 300,000 International Students Plan. Culture, Sports, Science and Technology.

7. Japan Ministry of Justice (2013) Points-based preferential immigration treatment for highly skilled foreign professionals. Immigration Bureau.

8. Kuwano N, Fukuda H, Murashima S (2016) Factors affecting professional autonomy of Japanese nurses caring for culturally and linguistically diverse patients in a hospital setting in Japan. J Transcult Nurs 27: 567-753.

9. Fujiwara Y (2008) Culturally congruent care: a concept analysis. J Jap Acad of Midwif 22: 7-16. 
10. Huang YL, Yates P, Prior D (2009) Factors influencing oncology nurses' approaches to accommodating cultural needs in palliative care. J Clin Nurs 18: 3421-3429.

11. Tsujimoto C (2011) Medical Tourism ni okeru suishinsenryaku ni kansuru kousatsu. Japan Foundation for International Tourism 18: 49-54.

12. Korean Global Healthcare Association (2014) The Korean Ministry of Health and Welfare.

13. Park HS, Ha SJ, Park JH, Yoo JH, Lee SH (2014) Employment Experiences of Nurses Caring for Foreign Patients. J Korean Acad Nurs Adm 20: 281-291.

14. Braun V, Clarke V (2006) Using thematic analysis in psychology. Qual Res Psychol 3: $77-101$.

15. Esmaeili M, Cheraghi MA, Salsali M (2013) Barriers to patient-centered care: a thematic analysis study. Int J Nurs Knowl 25: 2-8.

16. Lincoln and Guba's evaluation criteria (2017) Qualitative Research Guidelines Project. Robert Wood Johnson Foundation.

17. Plonien C, Baldwin KM (2014) Medical tourism: a nurse Executive's need to know. AORN J 100: 429-434. [Crossref]

18. Festini F, Focardi S, Bisogni S, Mannini C, Neri S (2009) Providing transcultural to children and parents: an exploratory study from Italy. J Nurs Scholarsh 41: 220-227.
19. Adachi Y, Ogawa M, Satake N, Hizume Y, Mikawa M, et al. (2009) Problems in caring patients with different cultural background -a postal survey of public hospitals in Japan. Nursing Journal of Osaka University 15: 19-31.

20. Maeno M, Sakuyama M, Motoyama S, Matsuo H (2011) Japanese nurses' views of perioperative management of foreign patients in Osaka. J Int Health 26: 273-280.

21. Kawauchi K (2011) Present situation and problems of medical interpreters in Japan research of medical treatment for foreigners. Kyushu Communication Studies 9: 25-35.

22. Sellman D (2003) Open-mindedness: a virtue for professional practice. Nurs Philos 4 17-24. [Crossref]

23. Ono N (2007) Review of description of transcultural nursing in United States texts. SCU Journal of Design \& Nursing 1: 15-21

24. Ono S, Yamamoto Y (2011) Cultural competence in nursing: a literature review. Kawasaki Medical Welfare Journal 20: 507-512.

25. Nonaka C, Higuchi M (2010) The process of building relationships between foreign patients and nurses in Japan. J Int Health 25: 21-32.

26. Makimoto K, Adachi Y, Satake N, Hizume Y, Mikawa M (2005) Hospital preparednes for foreign patients: a postal survey of 97 public hospitals in Japan. Nursing Journal of Osaka University 11: 24-29.

27. Jirwe M, Gerrish K, Keeney S, Emami A (2009) Identifying the core components of cultural competence: findings from a Delphi study. J Clin Nurs 18: 2622-2634.

Copyright: (2017 Kuwano N. This is an open-access article distributed under the terms of the Creative Commons Attribution License, which permits unrestricted use, distribution, and reproduction in any medium, provided the original author and source are credited. 